During this funding period, the laboratories of Drs. Anderson and 9 fitifert 1eturago performed a considerable amount of work toward addressing the issues associated with small volume analysis necessary for single cell studies. In that respect, our research has been focused on (1) developing new assays that can be miniaturized and are suitable for small volume and single cell analysis; (2) fabricating pL-vials that simulate the volume of single cells and setting up instrumentation capable of low-volume detection; (3) developing reproducible and reliable microinjection techniques; (4) developing methods of analysis for biomolecules in the pL-vials and employing these assays in the detection of biomolecules in single cells. The accomplishments attained in all these areas are described below. A total of 24 publications and 35 presentations have resulted from this work.

\title{
Development of New Assays
}

The assay for biotin that was developed in Dr. Daunert's laboratory based on the bioluminescence of aequorin can detect attomole levels of the vitamin biotin in the $\mu \mathrm{L}$-scale (42). In order to be able to perform these experiments, the biotinylated aequorin that is employed in the development of the assay for biotin is prepared by chemical conjugation of biotin to the photoprotein. Since it is advantageous that the biotinylated aequorin used in the assay development has the same bioluminescence characteristics each time that is prepared, it is important to determine the number of biotin molecules that have been attached per protein molecule. For that, we developed a method that allows us to estimate the degree of biotinylation of a protein (64).

Likewise, we are pursuing a recombinant DNA-based strategy to prepare recombinant biotinylated aequorin, which ensures the highly reproducible preparation of a homogeneous population of the reagent (65). Investigations toward the development of assays for other biologically relevant molecules have also been undertaken in our laboratories. In that respect, assays are being developed that are capable of detecting peptides (66). In another project, the feasibility of using green fluorescent protein (GFP) as a label in binding assays was investigated. GFP is a $27-\mathrm{kDa}, 238$ amino acid, naturally fluorescent protein. It has a maximum absorption peak at $395 \mathrm{~nm}$ with a shoulder at $470 \mathrm{~nm}$ and emits green light at $507 \mathrm{~nm}$ and $540 \mathrm{~nm}(67)$. These fluorescence characteristics are due to the presence of an internal chromophore, formed by the post-translational oxidation of residues $\mathrm{Ser}^{65}, \mathrm{Tyr}^{66}$ and $\mathrm{Gly}^{67}$ of the protein (68-70). The crystal structure shows that GFP has a $\beta$-barrel motif, with the chromophore residing within the interior of the $\beta$-barrel (see Figure below) $(71,72)$. A binding assay for biotin using GFP as the label was demonstrated. A mutant of GFP that contains a six-histidine tail to facilitate purification on an immobilized metal affinity column was chemically modified to incorporate biotin moieties. By varying the biotin to GFP ratio used for the conjugation reaction, several conjugates were prepared. These conjugates retained the fluorescence characteristics of GFP and were evaluated for use in the development of assays for biotin. The biotin on GFP was recognizable by avidin, however, the avidin conjugate interaction caused no or minimal perturbation in the fluorescence of GFP. Therefore, these conjugates were not suited for the development of homogeneous fluorescence binding assays (required for intracellular analysis), but rather for heterogeneous assays. Such an assay was developed in our laboratory (73) in what we believe is the first demonstration of using GFP as a label in competitive binding assays. 


\section{DISCLAIMER}

This report was prepared as an account of work sponsored by an agency of the United States Government. Neither the United States Government nor any agency thereof, nor any of their employees, make any warranty, express or implied, or assumes any legal liability or responsibility for the accuracy, completeness, or usefulness of any information, apparatus, product, or process disclosed, or represents that its use would not infringe privately owned rights. Reference herein to any specific commercial product, process, or service by trade name, trademark, manufacturer, or otherwise does not necessarily constitute or imply its endorsement, recommendation, or favoring by the United States Government or any agency thereof. The views and opinions of authors expressed herein do not necessarily state or reflect those of the United States Government or any agency thereof. 


\section{DISCLAIMER}

Portions of this document may be illegible in electronic image products. Images are produced from the best available original document. 
A.

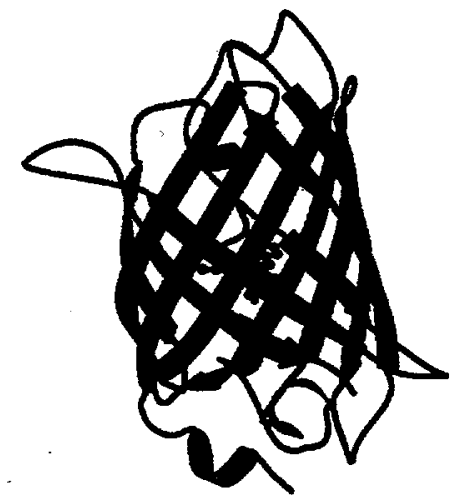

B.

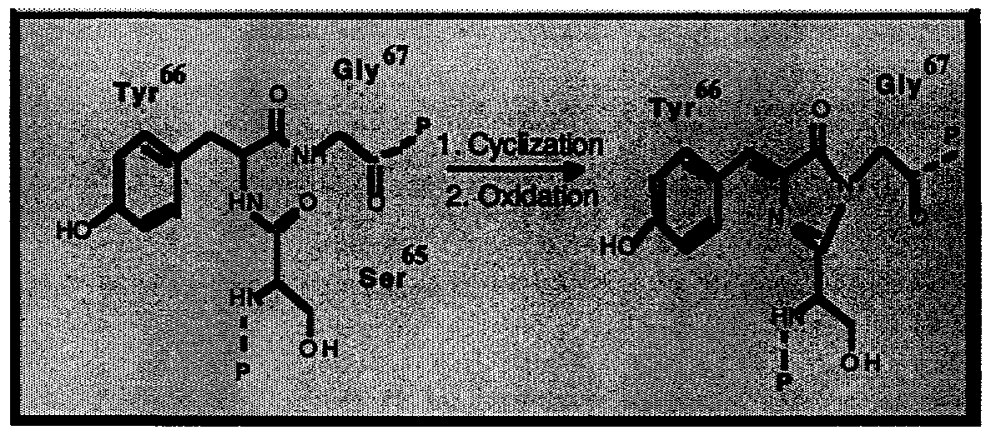

Figure 4. A. X-ray crystal structure of GFP. The internal chromophore is shown in balland-stick and lies within the b-barrel network of the protein. B. Formation of the chromophore in GFP from the amino acids $\mathrm{Ser}^{65}, \mathrm{Tyr}^{66}$ and $\mathrm{Gly}^{67}$. P denotes the rest of the protein sequence.

\section{Microfabrication and Instrumentation}

The goal of this project is to develop new methods capable of determining chemical components in small volumes and in single cells based on highly sensitive bioluminescence assays. In order to be able to perform these studies, we had to design and assemble instrumentation capable of monitoring cellular components within the small volume of a single cell. Therefore, our efforts were initially concentrated on identifying and purchasing the instrumentation necessary to perform our experiments. In that respect, Dr. Anderson's epifluorescence microscopy system was upgraded with a photon-counting photomultiplier detector connected to a data-acquisition system. In addition, Dr. Daunert purchased an inverted epifluorescence microscope and a highly sensitive photon-counting photomultiplier detector connected to a data-acquisition system partly with DOE funds. This instrumentation can be configured both in an epifluorescence mode and in a chemi(bio)luminescence mode.

At the same time that we were assembling the instrumentation in both laboratories, we started devising a method that would allow us to perform the assays in volumes comparable to those of single cells. The assay for biotin that was developed in Dr. Daunert's laboratory based on the bioluminescence of aequorin (see above) needed to be scaled down to $\mathrm{pL}$-volumes. In order to be able to develop assays at the $\mathrm{pL}$ scale, we needed optically transparent vials capable of holding picoliter-volume samples. Given that picoliter sample vials are not commercially available, we designed an array of picoliter vials on an optically transparent silica wafer that was fabricated at the National Nanofabrication Facility (NNF) at Cornell. Initial studies indicated that the wafer could satisfy the optical transparency properties desired. Vials used in these studies were fabricated using photolithographic procedures, which typically require many steps and a number of reagents. While the time required to produce vials is reduced by the reuse of a template, changes in the design of the vials require that the whole process be repeated. Thus, we decided to use a simpler and more versatile procedure, namely laser ablation, for the fabrication of our picoliter vials. 
The narrow width of a laser beam can "drill" picoliter vials into glass microscope coverslips with a wide range of depth and width without need of mask preparation. These vials prepared in collaboration with Dr. Janet Lumpp, from the Department of Electrical Engineering of the University of Kentucky are currently being used in all our experiments. The picoliter vials are fabricated by laser ablation using an excimer $\mathrm{KrF}$ laser at $248 \mathrm{~nm}$. This type of laser was chosen due to its ease of operation, high energy output per photon, and flat beam profile. The vials fabricated were carefully characterized in terms of reproducibility in the manufacturing with respect to width and depth by using a stylus profilometer. The diameter of the picoliter vials was $100 \mu \mathrm{m}$ ( $\mathrm{rsd}<3 \%, \mathrm{n}=8)$. It is important to note that the vials can be fabricated in a very reproducible manner. We have fabricated vials with diameters of $63 \mu \mathrm{m}$ (corresponding to $\sim 80 \mathrm{pL}$ ) and it is theoretically possible to fabricate vials with diameters as small as $\sim 17 \mu \mathrm{m}$ (corresponding volume of $\sim 2 \mathrm{pL}$ ). The depth of the picoliter vials is controlled by the number of pulses used to ablate each vial. The vials being used in our studies have depths of $\sim 45 \mu \mathrm{m}$, which can be obtained with 350 laser pulses with a laser power of $26 \mathrm{~mW}$ (measured at the source). The relative standard deviation of the depth of vials machined on a given day is $<2 \%$ (n $=4)$. Vials having a diameter of $100 \mu \mathrm{m}$ and a depth of $45 \mu \mathrm{m}$ making for a total volume of 350 $\mathrm{pL}$ are shown in Figure 5.

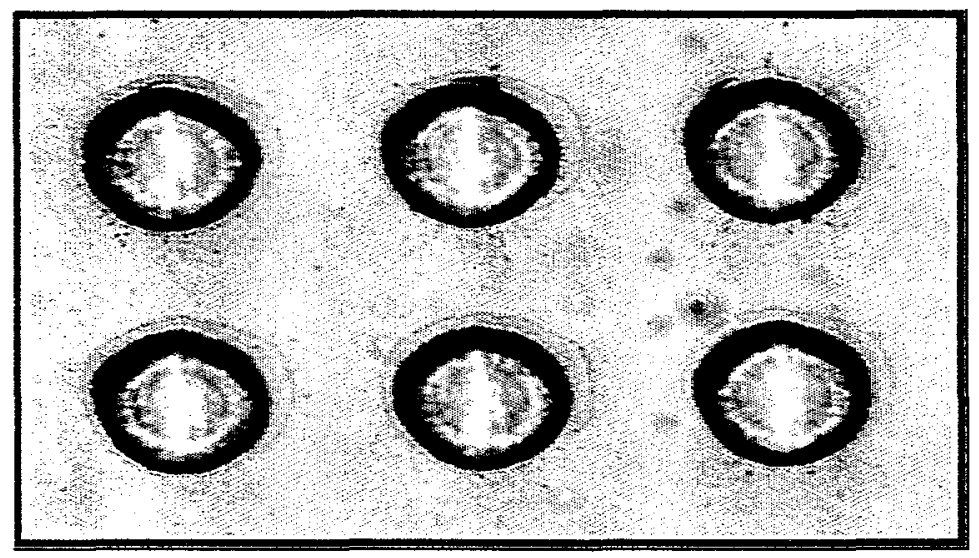

Figure 5. An array of vials fabricated using the laser ablation technique.

\section{Reproducible and Reliable Microinjection Techniques}

In order to develop reliable analytical techniques for microanalysis, the issue of reproducibility in the delivery of the reagents is critical. Although the manufacturers of commercial microinjectors claim that volumes of less than $1 \mathrm{pL}$ can be injected, there is a lack of experimental data showing that such low volume injections can be made reproducibly. Since reproducibility of pL-volume injections plays a key role in the development of our assay, it was necessary to develop a new method for calibrating micropipets, and determining accurate injection volumes using a commercial pressure-based microinjector in conjunction with the 


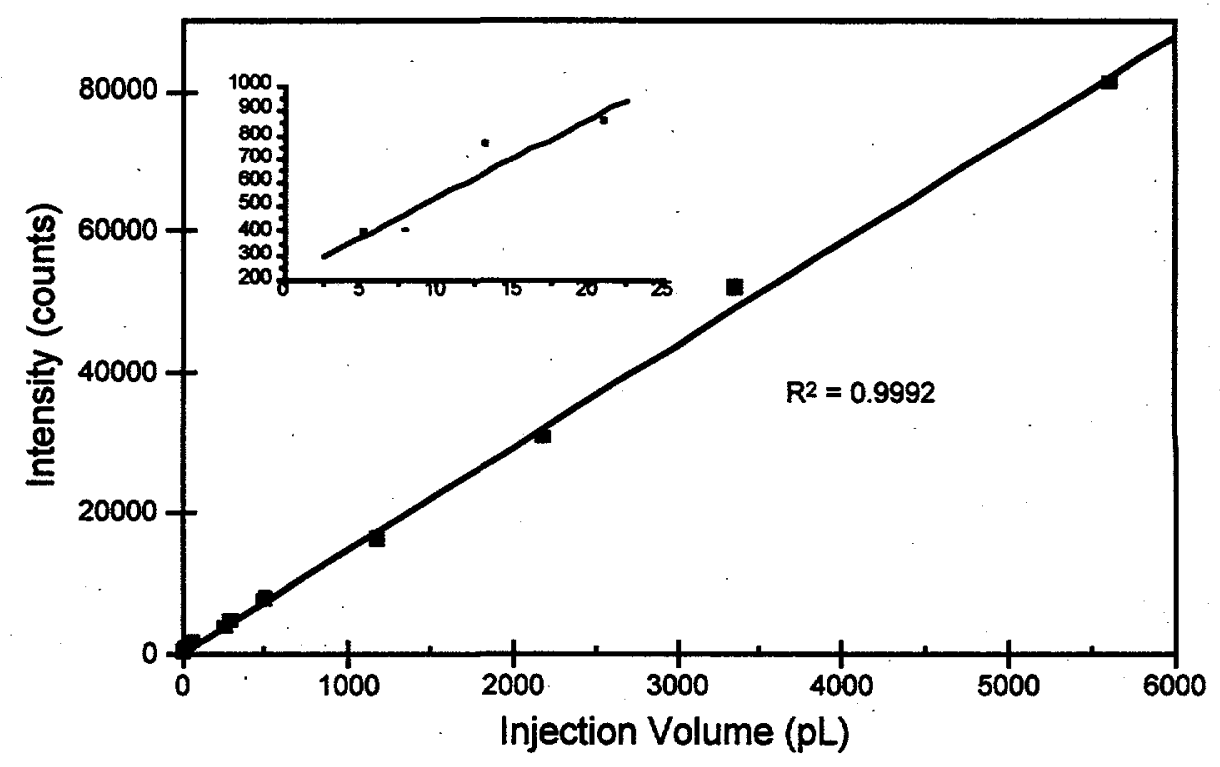

Figure 6. Calibration plot for aequorin in the pL-vials.

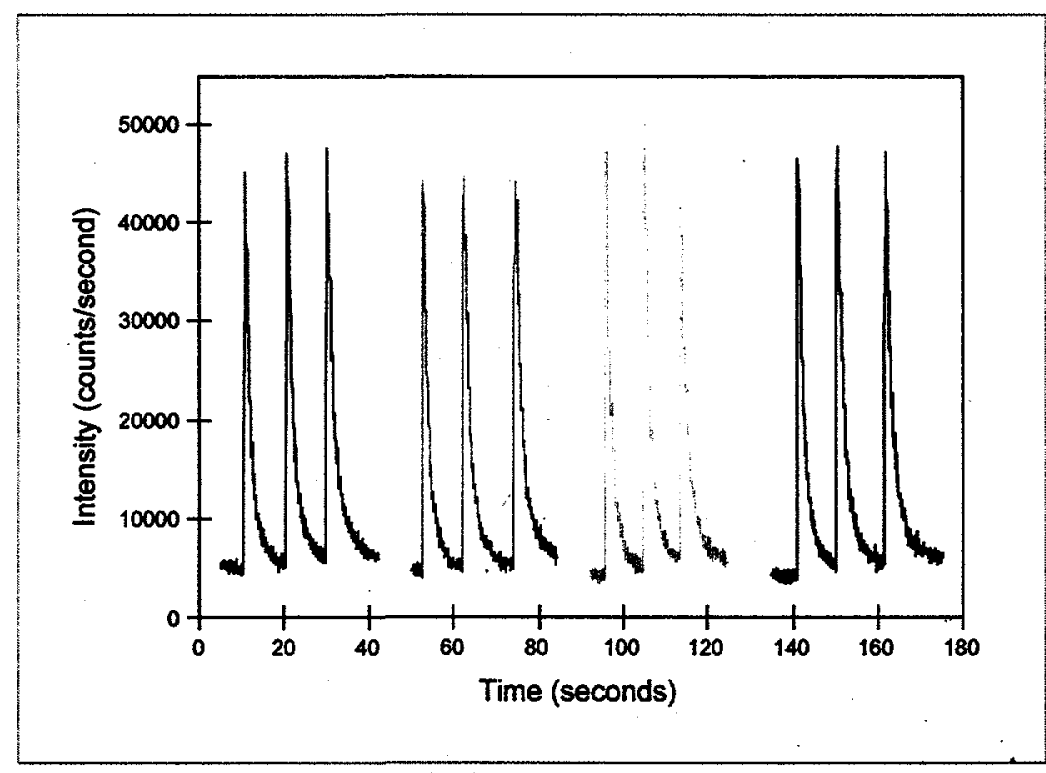

Figure 7. Reproducibility of the injection of biotinylated aequorin into four different $\mathrm{pL}$ vials.

bioluminescent protein aequorin. In contrast to conventional micropipet calibration methods that employ fluorescent molecules, in the present method the luminescence background is much smaller than those seen using fluorescent probes. This method can be used to determine injection volumes as small as $3 \mathrm{pL}$, and the calibration plots are linear over at least three orders of magnitude (see Figure 6 above). These data are described in detail in reference 4 of the Progress Report.

Figure 7 shows the bioluminescence of aequorin in four different vials. The reproducibility of the signals obtained for multiple injections of $200 \mathrm{amol}$ of biotinylated 
aequorin into a series of vials is depicted in the Figure. Each set of three injections was made in a different vial. For injections into a single vial the relative standard deviation of peak areas was $<8 \%$ in all cases. The relative standard deviation of the peak areas from all twelve injections was $4.3 \%$, which shows that the injection of aequorin and the detection of the resulting signal is reproducible from vial to vial.

\section{Methods of Analysis in pL-Volumes and in Single Cells}

The development of a reliable and reproducible method of injection of $\mathrm{pL}$-volumes of reagents along with the successful microfabrication of $\mathrm{pL}$-vials has been a significant step toward the development of our assays. By employing these vials and our method of delivering reagents, we have been able to prepare calibration plots for the detection of aequorin down to 5 amol. It is important to note that we can observe the flash-kinetics that are characteristic of the bioluminescence of aequorin even at these very low levels of the protein (Figure 8). We have

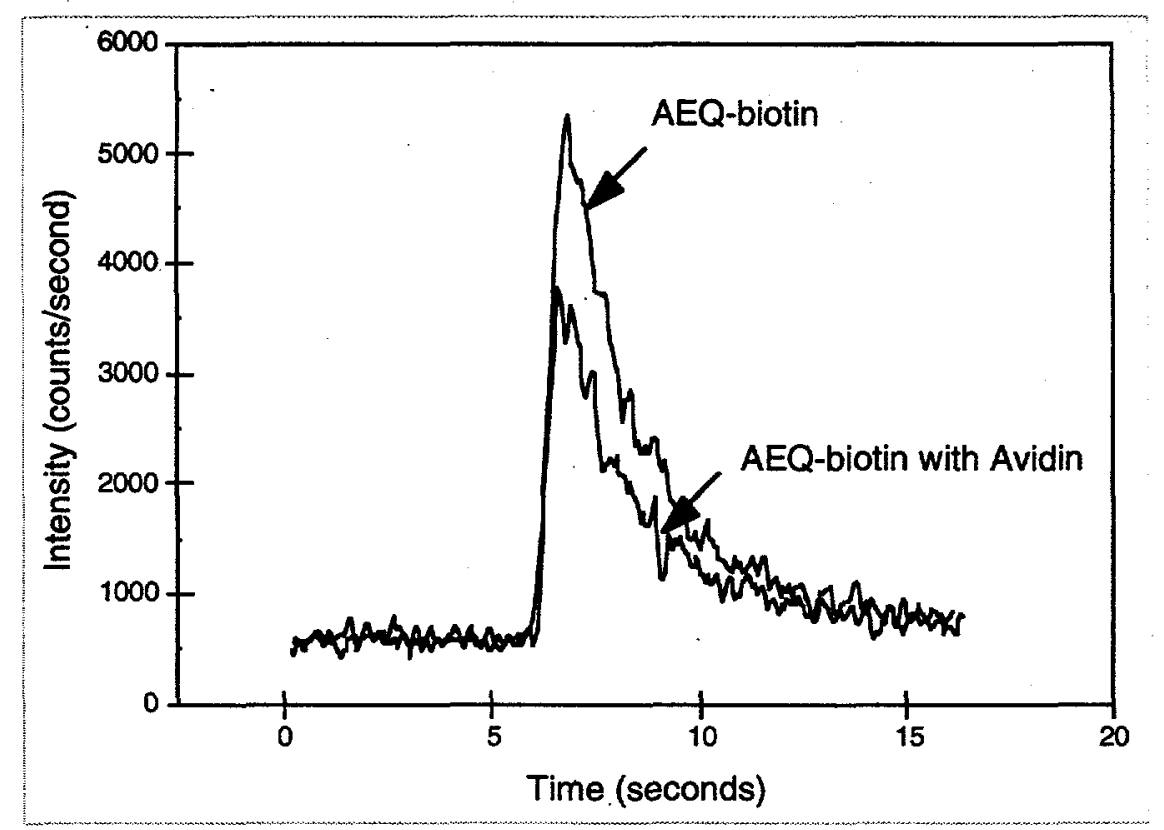

Figure 8. Inhibition of bioluminescence signal of biotinylated aequorin by avidin in the picoliter-volume vials.

previously demonstrated using a million-fold larger volumes (in the $300 \mu \mathrm{L}$ range) that when avidin binds to biotinylated aequorin, the bioluminescence emission of aequorin-biotin is inhibited (42). In order to develop a binding assay for avidin in the picoliter vials, the amount of biotinylated aequorin needs to be optimized so that it is large enough to give a significant signal, but as small as possible. This is because in binding assays such as this, better limits of detection are obtainable with smaller amounts of the labeled ligand. Therefore, the amount of AEQ-biotin chosen for further studies was $10^{-16} \mathrm{~mol}$. In these experiments, avidin was injected first into picoliter vials followed by injection of the AEQ-biotin. Then, the bioluminescence was triggered by injecting $100 \mathrm{pL}$ of a buffered $\mathrm{Ca}^{2+}$ solution. Figure 8 shows that there is a clear decrease in the bioluminescence signal generated by $10^{-16} \mathrm{~mol}$ of AEQ-biotin in the presence of avidin. 
Incubation time is crucial in conventional binding assays (i.e., in microliter-scale experiments). We hypothesized that the picoliter-scale experiments would not require the same 30 -min incubation time as in the $\mu \mathrm{L}$-scale experiments (42) because of the limited volume of the picoliter vials, the much higher concentrations used, and the mixing produced by the injection process. A series of experiments where a solution containing both calcium and avidin was added to picoliter vials containing AEQ-biotin was conducted to confirm that AEQ-biotin binding to avidin was rapid. In these experiments, the expected inhibition of AEQ-biotin bioluminescence by avidin was seen. Thus, incubation of AEQ-biotin and avidin before triggering the aequorin bioluminescence with $\mathrm{Ca}^{2+}$ is not necessary since the association between these two biomolecules is extremely rapid in the picoliter vials. However, in practice, the AEQ-biotin and avidin were incubated while the calcium pipet was mounted and positioned.

A calibration plot for avidin was prepared by keeping the concentration of biotinylated aequorin in the pL-vials constant and varying the amounts of avidin injected to the vial (Figure 9).

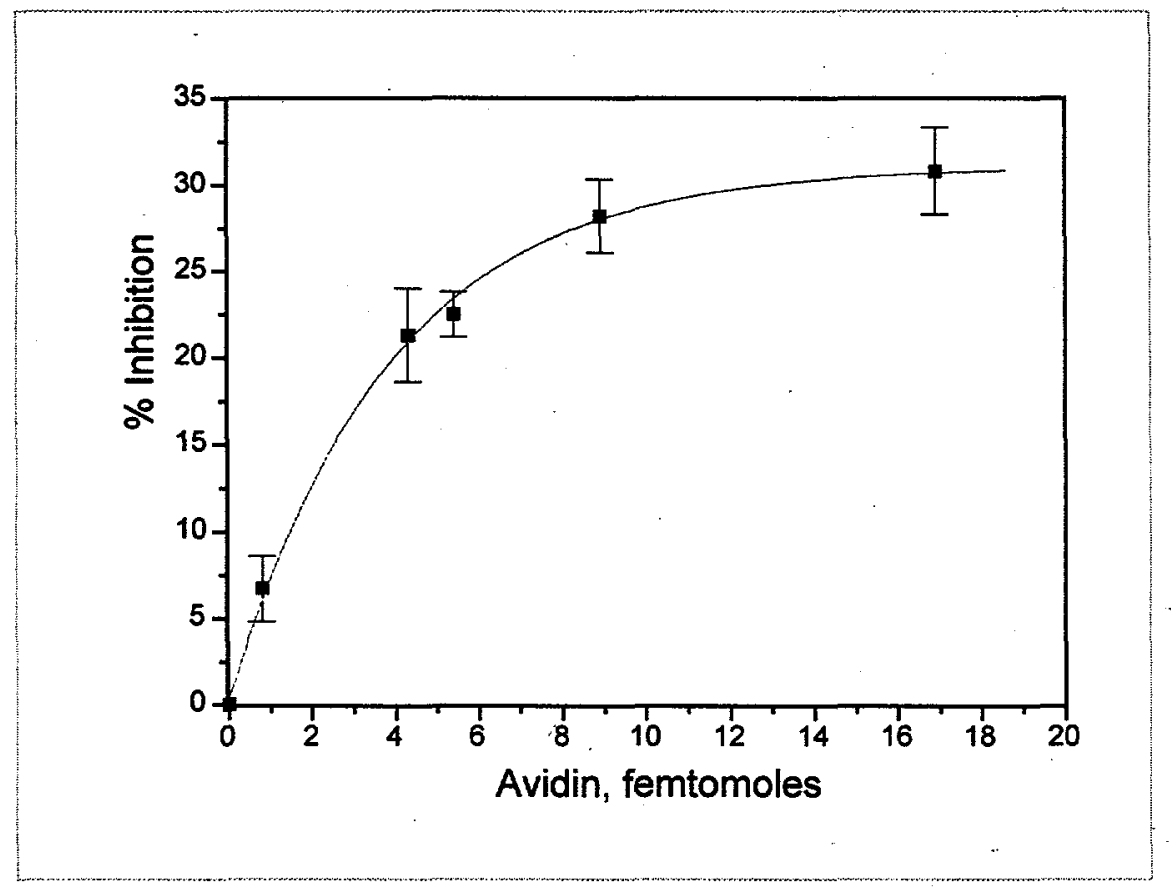

Figure 9. Calibration plot for avidin in the picoliter-vials. This is a plot of the inhibition of the bioluminescence signal of biotinylated aequorin in the presence of different amounts of avidin.

This figure indicates that amounts of avidin as low as $1 \times 10^{-15} \mathrm{~mol}$ can be detected in the picoliter vials. The maximum inhibition of biotinylated aequorin observed when an excess of avidin was added was $\sim 30 \%$. The same level of inhibition was observed with a conventional luminometer when avidin and biotinylated aequorin were allowed to equilibrate $30 \mathrm{~min}$ before triggering bioluminescence with buffered calcium solution. This is quite remarkable if one takes into account that we are working on a $10^{6}$-fold smaller scale. The reproducibility of our results is also very good, with relative standard deviations ranging from $2-13 \%$. 
A competitive binding assay for biotin was developed in the pL-vial scale by using the arrays of vials fabricated by laser ablation. A typical calibration curve for biotin in the $\mathrm{pL}$-vials is shown in Figure 10 below. This is the first time that a competitive binding assay has been achieved in a picoliter-volume vial. A sequential assay was also performed in the pL vials. As seen in Figure 11, the curve is linear over a larger range, but does not have the same detection limit as the competitive assay. Using this assay as a model, individual sea urchin oocytes were injected with AEQ-biotin and AEQ-biotin plus avidin, and the relative bioluminescence signal was obtained. Figure 12 shows the distribution of values obtained for one day's experiments. The average signal from two separate sets of oocytes taken on separate days had average relative signals of $0.803(n=6)$ and $0.775(n=10)$. By comparing these data to the dose response curve in Figure 11, this corresponds to an average of 4 and 5 femtomoles, respectively. It should be noted that these data have a very wide standard deviation, as, indeed, we would expect from a heterogeneous population of cells, and is the case in Figure 12.

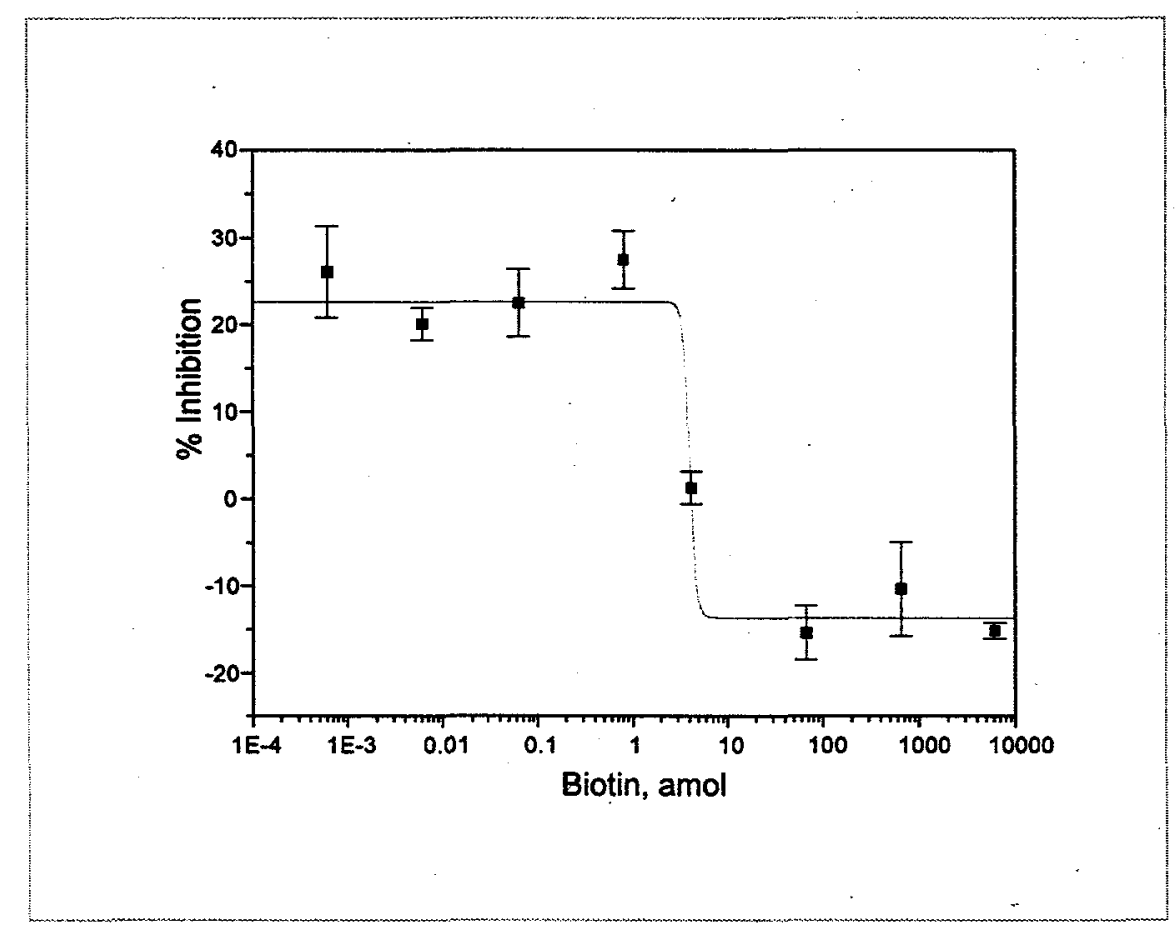

Figure 10. Competitive calibration curve for biotin performed in the $\mathrm{pL}$-volume vials. All experiments used a fixed volume of premixed biotinylated aequorin and different concentrations of biotin. These solutions were injected into a vial containing a solution of avidin with $\mathrm{Ca}^{2+}$. 


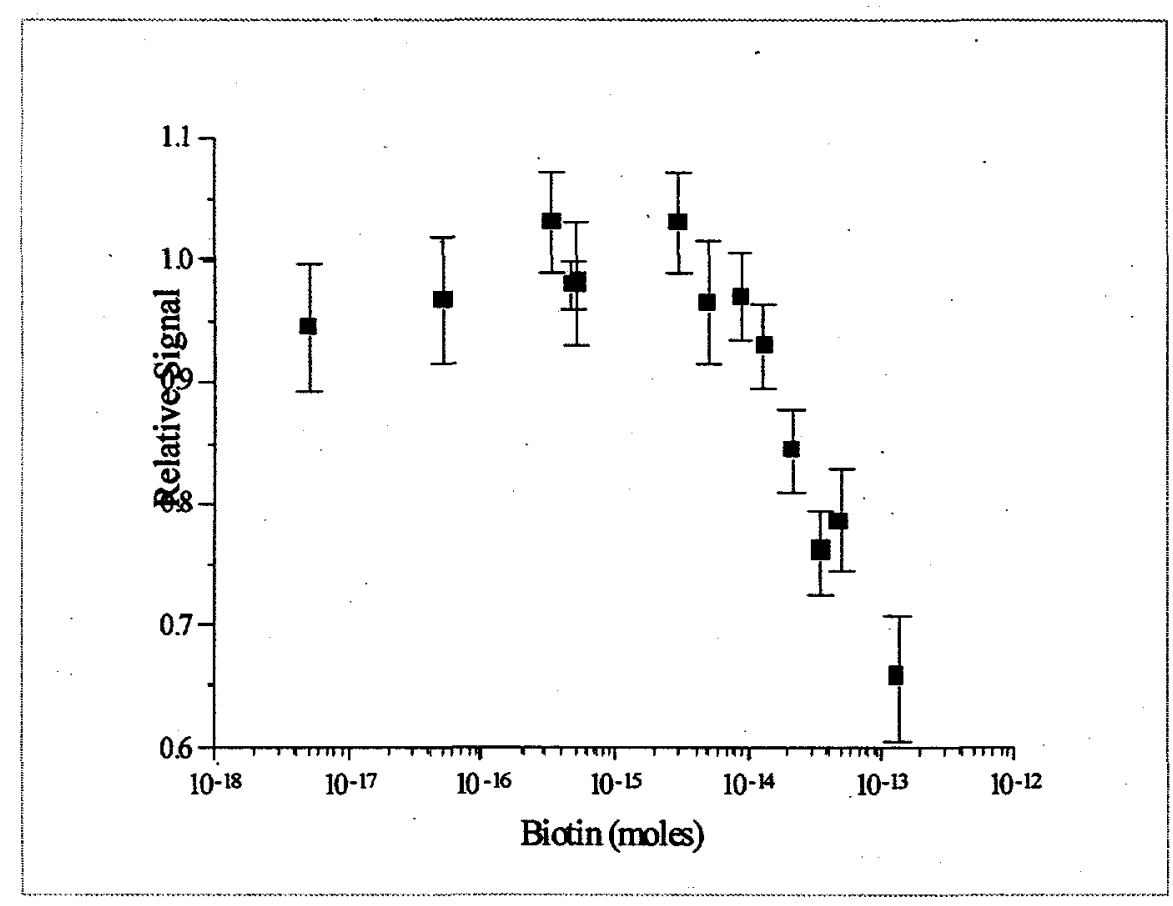

Figure 11. Sequential dose-response curve for biotin in the $\mathrm{pL}$ vials, in which biotinylated aequorin, avidin, and biotin, were added separately.

The oocytes were collected from a female sea urchin by injection with $0.5 \mathrm{M} \mathrm{KCl}$, so that the age of the individual oocytes, as well as their nutritional environment upon maturation, would be considerably different

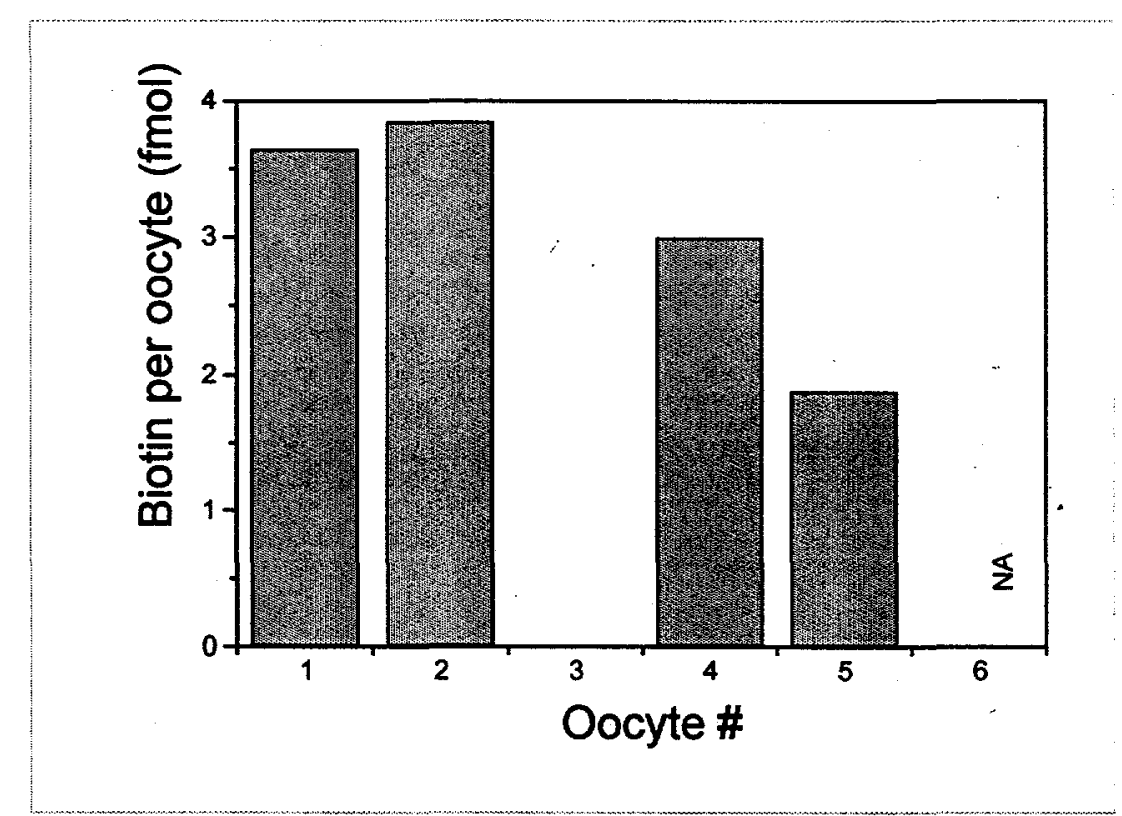

Figure 12. Histogram showing the distribution of amounts of biotin per oocyte. Results are from one day's experiments $(n=6)$. Oocyte number 3 has an amount of biotin below the linear range of the assay. Oocyte 6 shows an inhibition higher than those in the doseresponse curve. 
We also compared the luminescent signal of AEQ-biotin between samples containing mammalian cells (as opposed to the sea urchin oocytes), namely CREF cell lysate to controls in the picoliter vials. Results from the pL-vials indicated that there was a significant difference in the luminescence intensity between samples containing cells and samples not containing cells. Specifically, samples containing cells showed a smaller inhibition of the bioluminescence signal than those containing simply AEQ-biotin and avidin, which is consistent with the presence of biotin in these samples. These results can be seen in Figure 13.

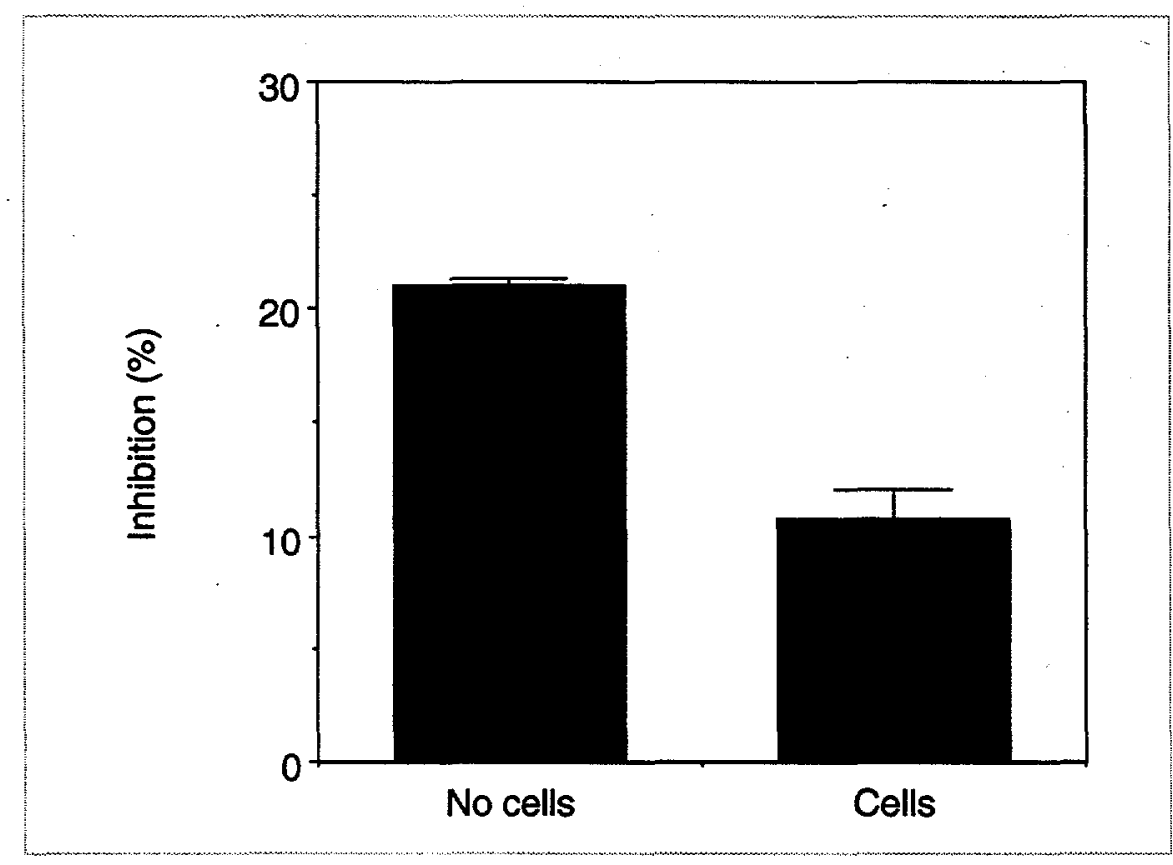

Figure 13. Comparison of inhibition in $\mathrm{pL}$-vials between samples containing lysate of CREF cells and those not containing CREF cells. All pL-vials contained AEQ-biotin and avidin at the same concentration. Black bars are results in one array of $8 \mathrm{pL}$-vials, while white bars are in another array. Errors are result of $n=4$ replicates.

Another experiment was performed using a fluorescently-labeled phosphate-binding protein (PBP). Previous experiments showed that this PBP conjugate experienced a maximum $93 \%$ enhancement in fluorescence when bound to phosphate. The amount of,enhancement was shown to be proportional to the amount of phosphate added. To test whether assays based on this theory could work in a $\mathrm{pL}$ vial, various concentrations of phosphate were preincubated with the labeled PBP before injection into a microvial. Injection volumes were on the order of 500 pL. The resulting dose-response curve is shown below.

In another experiment we show the utility of using the fluorescence assays in the pLvials. One assay developed in our laboratories depends upon the quenching of a fluorescently labeled calmodulin by phenothiazines. As seen in Figure 15, when this assay is performed in a spectrofluorometer or a microtiter plate reader, the detection limit is actually worse in terms of mass than in the epifluorescence microscope. So, by reducing the volume of the analysis, we gain the advantage of using less reagent, as well as the ability to detect fewer molecules of the 
analyte. This demonstrates that, not only can fluorescence assays be successfully used in microvolumes, they can possess superior response characterisics.

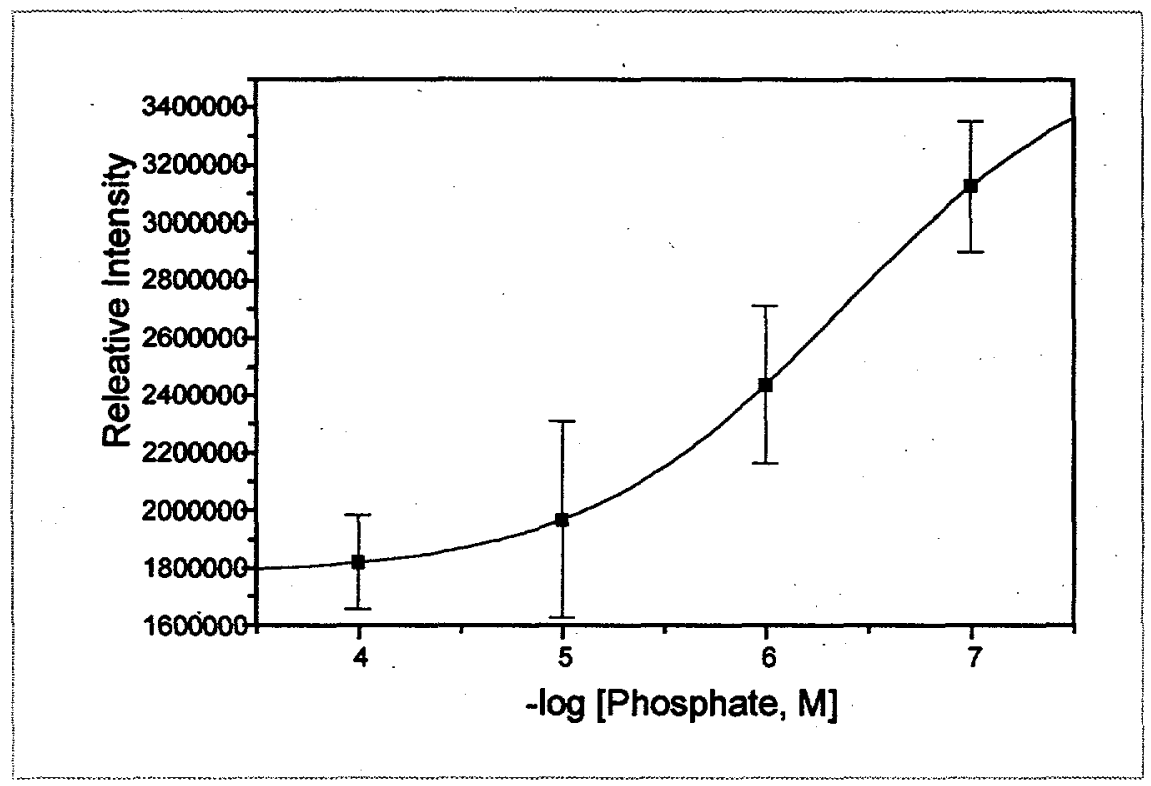

Figure 14. Dose-response curve for phosphate in the pL-vials. The detection limit is approximately $1 \times 10^{-7} \mathrm{M}$.

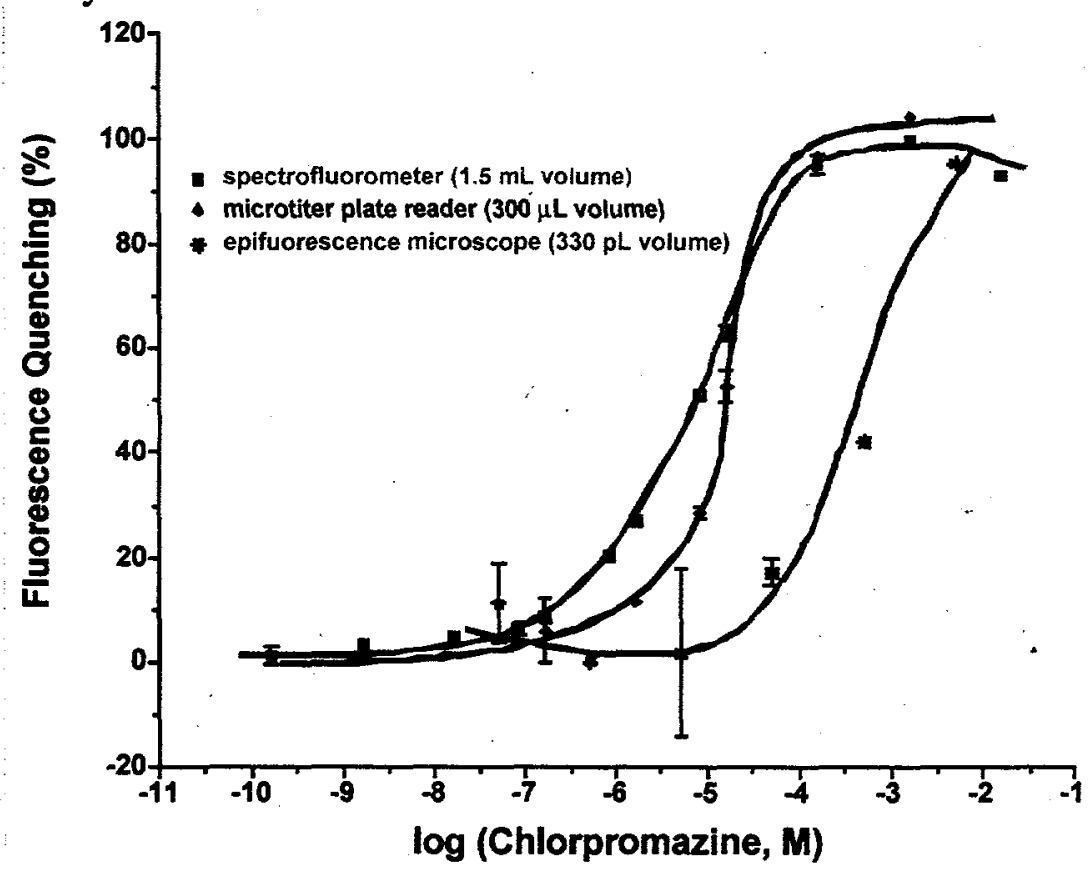

Figure 15. Comparison of the fluoresence assay for chlorpromazine (phenothiazine) in three different volumes. The response characteristics are the same in each case, with the curve being shifted to higher concentrations at lower volumes. The detection limit in the vials is $33 \mathrm{fmol}$ versus $300 \mathrm{pmol}$ in the microtiter plates and $150 \mathrm{pmol}$ in the spectrofluorometer. 
In order to compare the utility of the microinjection-based technique with other small volume assay techniques, a postcapillary reaction detection system for capillary electrophoresis (CE) was developed. Briefly, the sample was separated by capillary zone electrophoresis and the effluent stream was coupled to a binding assay specific for biotin and its analogues (e.g., biocytin) through a postcapillary reactor. The binding assay depends upon the increase in fluorescence of a streptavidin-fluorescein isothiocyanate conjugate (streptavidin-FITC) upon biotin binding. The degree of fluorescence enhancement is directly dependent upon the concentration of biotin in the sample (74). Thus, a biotin peak in the effluent stream will cause an increase in the fluorescence intensity of the streptavidin-FITC, with the peak area being related to the amount of biotin present.

A schematic of the CE apparatus is seen in Figure 16. After injection into the capillary, sample components are separated on the basis of charge-to-mass ratio in the separation capillary.

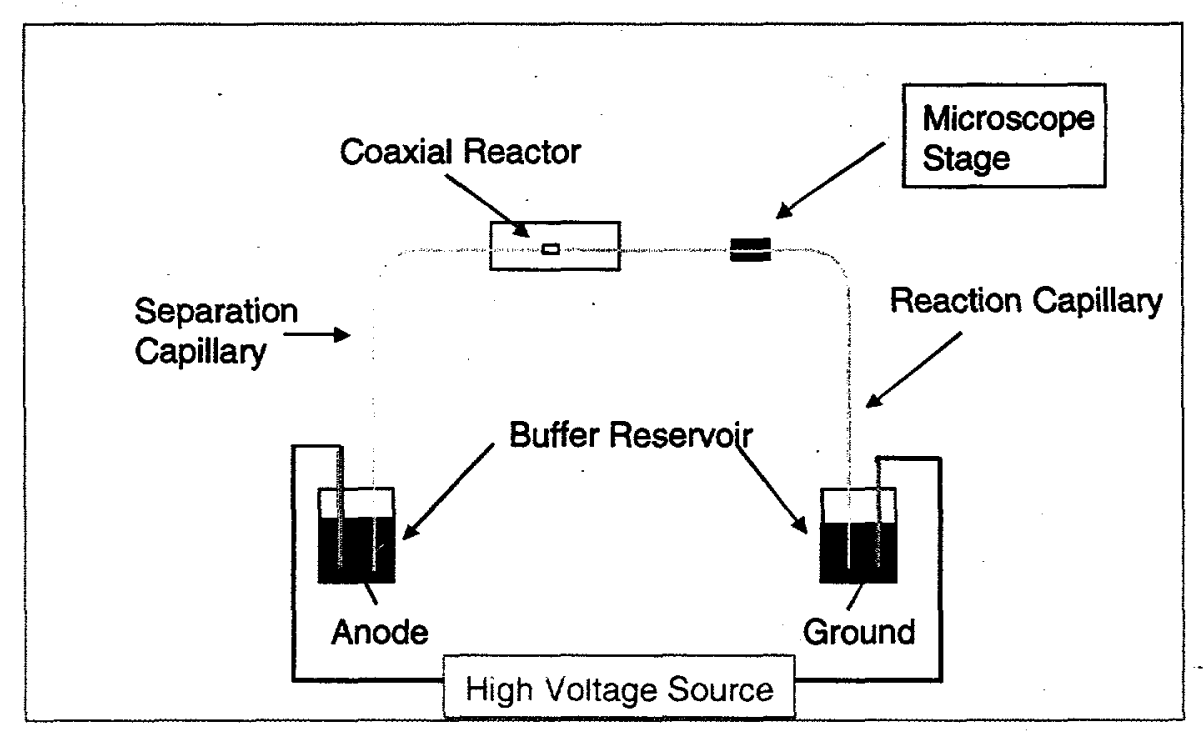

Figure 16. Schematic of the CE setup. The potential is supplied to the capillaries by means of platinum electrodes immersed in the buffer reservoirs. The reagent is siphoned into the reactor as shown in the close up view in Figure 12. The sizes of the separation and reaction capillaries are not drawn to scale.

The effluent of the separation capillary then enters the coaxial reactor (see Figure 17 for details),

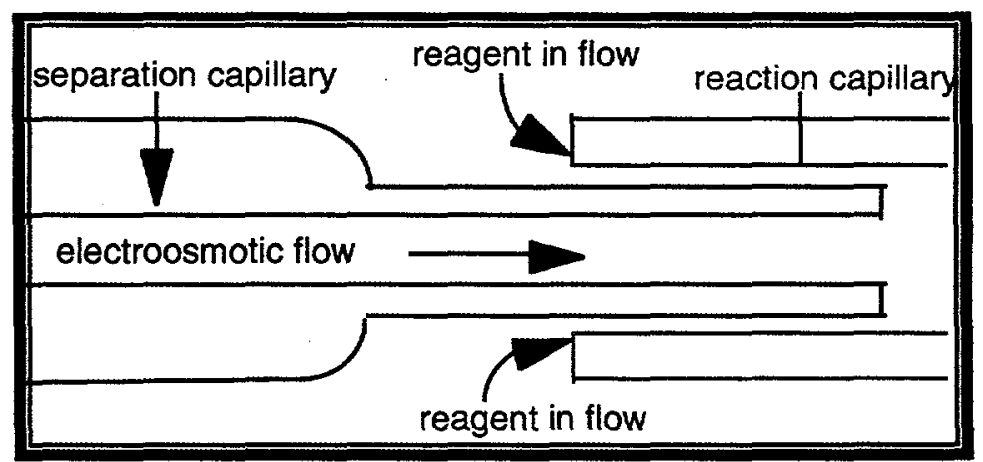

Figure 17. Close-up view of the coaxial reactor seen in Figure 11. 
where streptavidin-FITC is merged with the effluent. The combined stream is carried to the detector by electroosmotic and gravity flow. The separation capillary was a bare fused silica capillary of dimensions $77 \mu \mathrm{m}$ i.d. $\times 30 \mathrm{~cm}$ long. The reaction capillary was $144 \mu \mathrm{m}$ i.d. $\times 20 \mathrm{~cm}$ (length to detector). The reactor was constructed by removal of $1-\mathrm{cm}$ of coating on the separation capillary and etching of that capillary in concentrated HF. The etched separation capillary was then inserted into the reaction capillary. After the separation of biotin and biocytin had been optimized, a calibration curve for biotin and biocytin was completed. A typical electropherogram under the given conditions is seen in Figure 18. The calibration curve under the

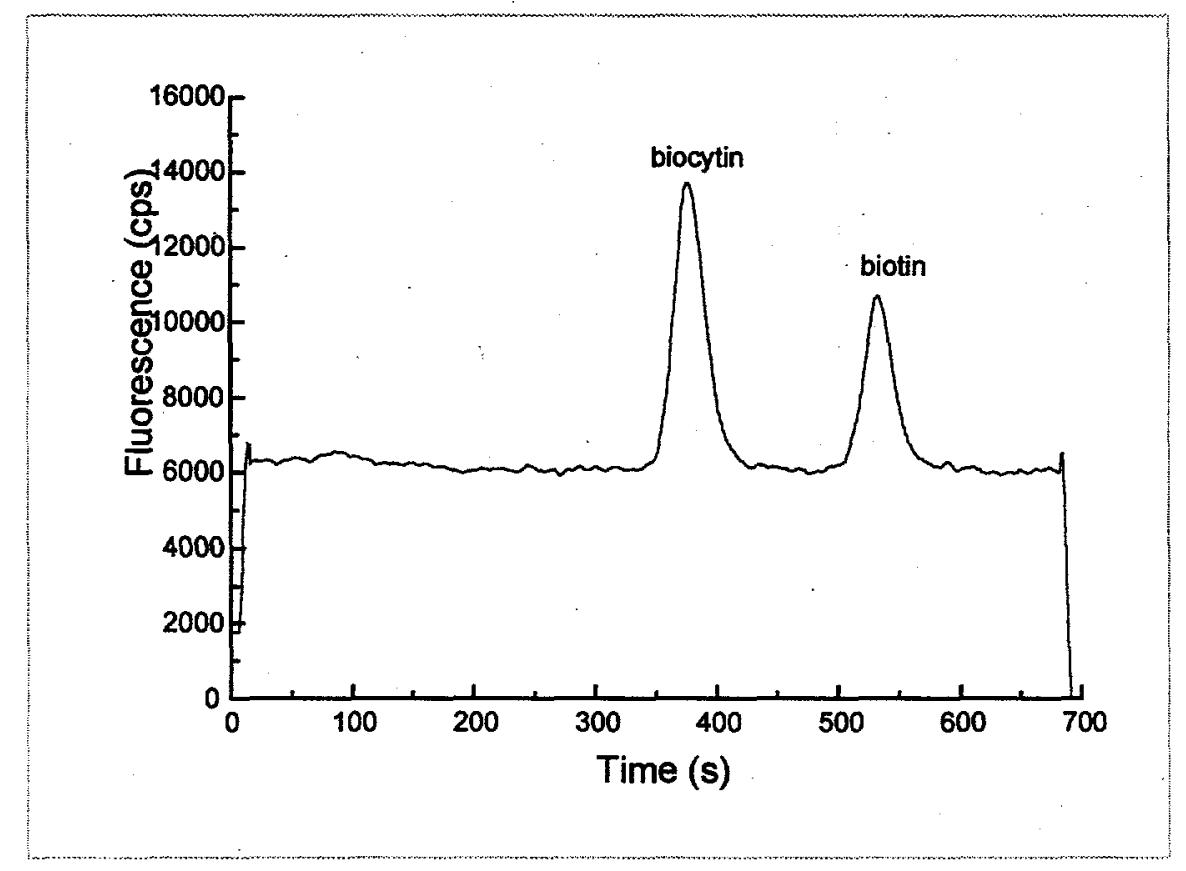

Figure 18. Typical electropherogram for the $\mathrm{CE}$ setup. Conditions are: running buffer, $30 \mathrm{mM}$ sodium phosphate, $\mathrm{pH} 7.5$; applied voltage, $-15 \mathrm{kV}$; streptavidin-FITC $1 \mu \mathrm{g} / \mathrm{mL}$; biotin/biocytin, $10 \mu \mathrm{M}$; injection time, $5 \mathrm{~s}$.

same conditions is seen in Figure 19. The response characteristics show a linear calibration curve for biotin between 0.1 and $100 \mu \mathrm{M}$, with a detection limit of $0.1 \mu \mathrm{M}$. As the estimated injection volume (based on a hydrodynamic injection of $5 \mathrm{~s}$ at a height of $30 \mathrm{~cm}$ ) of $15 \mathrm{~nL}$, this corresponds to a mass detection limit of 1500 amol.

The amount of biotin in a crude lysate of sea urchin oocytes was then determined. First the eggs were lysed by boiling, then insoluble particles were removed by centrifugation. This clarified lysate was then injected directly onto the capillaries. The resulting electropherogram is seen below in Figure 20. By comparing these data to the calibration curve in Figure 17, the concentration of biotin and biocytin was determined to be $1.9 \mu \mathrm{M}$ and $5.0 \mu \mathrm{M}$, respectively. Given that the number of oocytes per injection is 16 , this corresponds to a total of $6.5 \mathrm{fmol}$ of biotin-containing compounds per oocyte. 


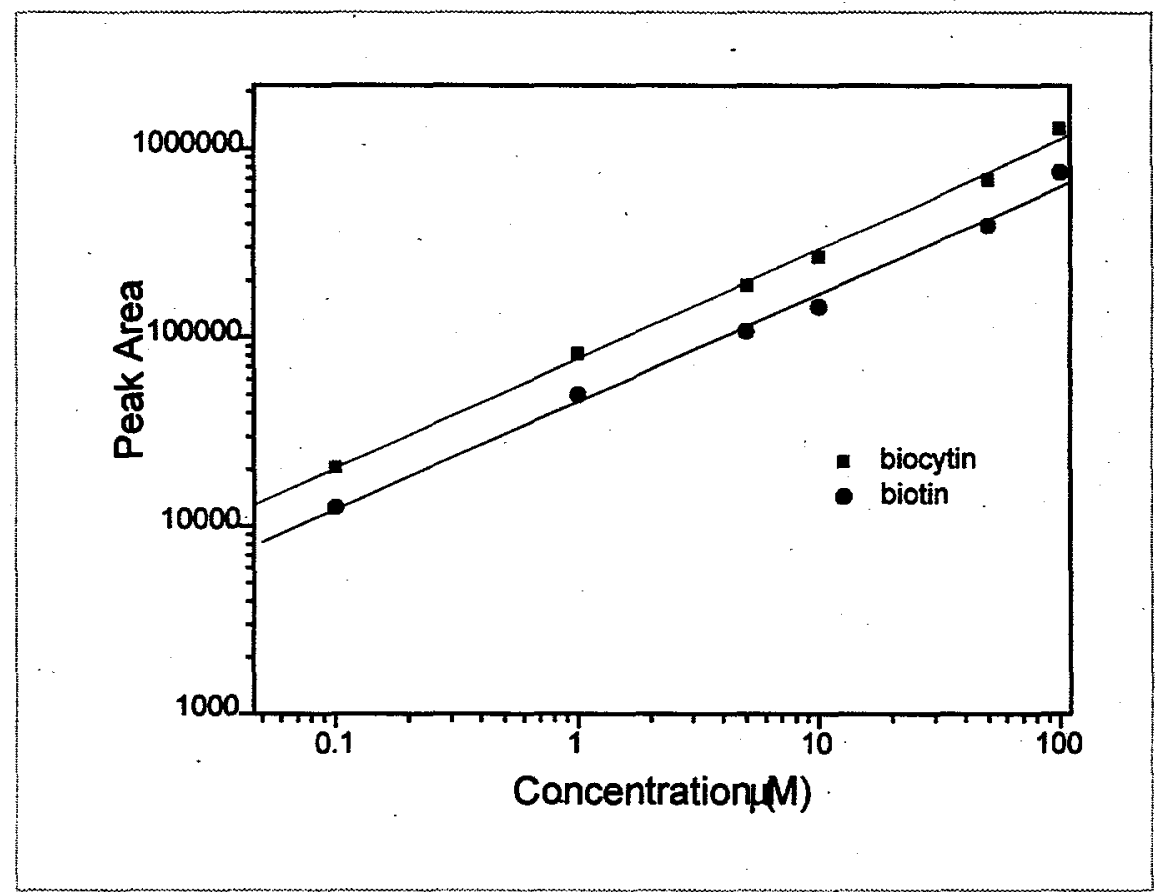

Figure 19. Calibration curve for biotin. Conditions are the same as in Figure 16. Each point is the average for each concentration \pm one standard deviation $(n=3)$.

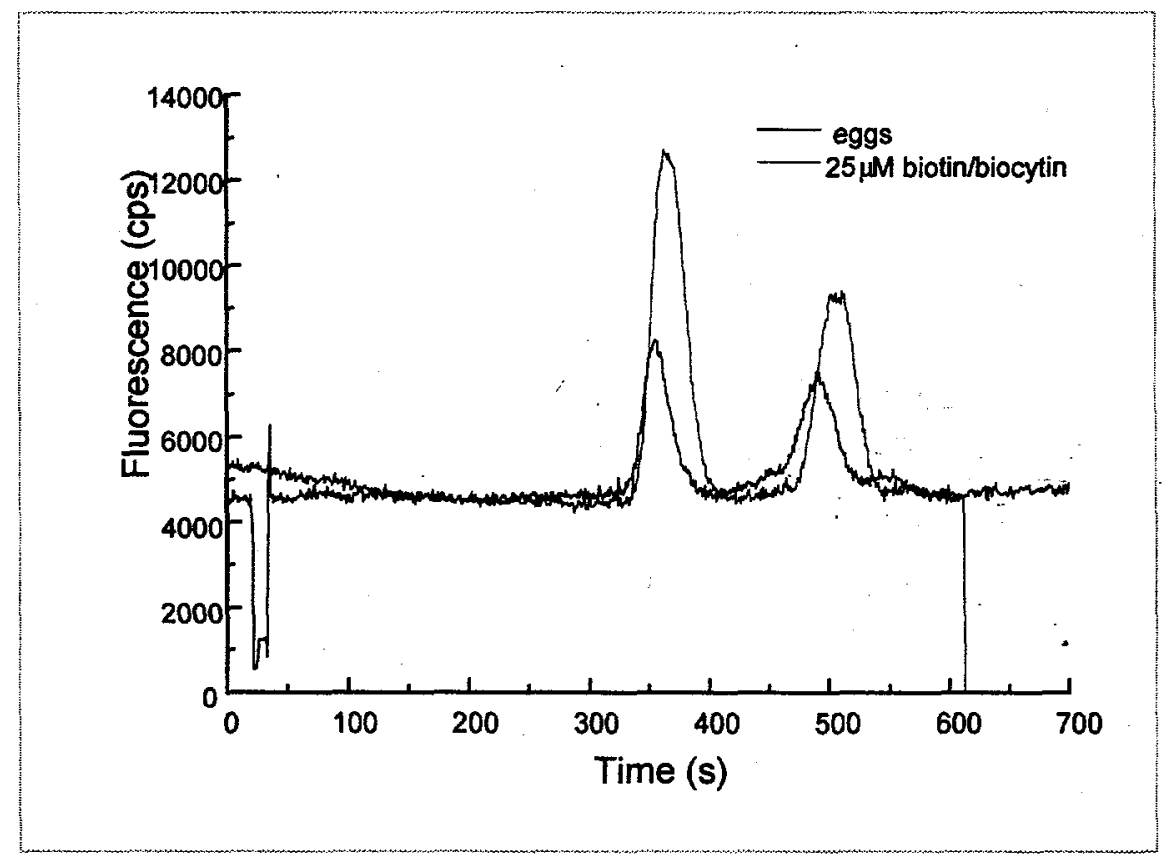

Figure 20. Electropherograms of sea urchin oocyte lysate, and a standard solution of 25 $\mu \mathrm{M}$ biotin and biocytin.

The results of this work indicate that we have developed techniques that are capable of detecting biotin in single cells. In the case of the sea urchin oocytes, the average amount of biotin detected using the aequorin-based assay $(4.5 \mathrm{fmol})$ is statistically not significantly 
different from that determined by using the capillary electrophoresis based assay ( $6.5 \mathrm{fmol}$ ). This indicates that the microinjection assay is accurate within a cell. It should be noted that the $\mathrm{CE}$ assay should not be prone to matrix effects, as it is unlikely that any contaminating species would have the same electrophoretic mobility as the biotin and biocytin. The CE sample was also prepared by completely lysing the cells, so that the total biotin contents of the cells should be seen. The aequorin assay thus must also be able to detect most, if not all, of the total cells contents, at least as far as biotin is concerned. These are important results in our continuing drive to develop assays for single cell analysis.

Presentations and Publications:

A total of 35 scientific presentations and the following publications and patents have resulted from research funded by this DOE Award:

PUBLICATIONS:

1. S. Lizano, S. Ramanathan, A. Feltus, A. Witkowski, and S. Daunert, "Bioluminescence Competitive Binding Assays for Biotin Based on the Photoprotein Aequorin", Methods Enzymol., 279, 296-303 (1997).

2. A. Grosvenor, C. Crofcheck, K. Anderson, D. Scott, and S. Daunert, "Calibration of Micropipets Using the Bioluminescent Protein Aequorin", Anal. Chem., 69, 3115-3118 (1997).

3. A. Feltus, S. Ramanathan, and S. Daunert, "Cooperative Interaction of Immobilized Avidin with Aequorin-Biotin Conjugates: An Aequorin-Linked Assay for Biotin", Anal. Biochem., 254, 62-68 (1997).

4. C. Crofcheck, A. Grosvenor, K. Anderson, J. K. Lumpp, D. Scott, and S. Daunert, "Detecting Biomolecules in pL-Vials Using the Bioluminescence of Aequorin", Anal. Chem., 69, 47684772 (1997).

5. S. Ramanathan, J. Lewis, M. S. Kindy, and S. Daunert, "Heterogeneous Binding Assay for an Octapeptide Based on a Fusion Protein with Recombinant Aequorin", Anal. Chim. Acta, ... 369, 181-188 (1998).

6. E. C. Hernández and S. Daunert, "Green Fluorescent Protein as a Novel Label in Binding Assays", Anal. Biochem. 261, 113-115 (1998).

7. J. C. Lewis, A. Feltus, M. C. Ensor, S. Ramanathan, and S. Daunert, "Applications of Reporter Genes in Analytical Chemistry", Anal. Chem., 70, 579A-585A (1998).

8. S. Matveev, J. C. Lewis, and S. Daunert, "Genetically Engineered Obelin as a Bioluminescent Label in an Assay for a Peptide", Anal. Biochem., 270, 69-74 (1999).

9. J. C. Lewis, and S. Daunert, "Dual Detection of Peptides in a Fluorescence Binding Assay by Employing Genetically Fused GFP and BFP Mutants", Anal. Chem., 71, 4321-4327 (1999).

10. J. C. Lewis, J. Feliciano, and S. Daunert, "GFP-Peptide Fusions in the Development of Fluorescence Immunoassays for Small Peptides", Anal. Chim. Acta, 397, 279-286 (1999).

11. J. C. Lewis, A. J. Feltus, and S. Daunert, "Recombinant Photoproteins in the Design of Binding Assays", ACS Symposium Series, invited contribution, in press, 2000.

12. J. C. Lewis, J.-J. López-Moya, and S. Daunert, "Production of Aequorin Mutants with Unique Cysteine Residues Demonstrating Distinct Bioluminescence and Secondary Structure Charateristics, Part I", Bioconj. Chem., in press, 2000.

13. J. C. Lewis, L. C. Cullen, and S. Daunert, "Preparation and Characterization of Aequorin Mutants and their Use in the Development of an Homogeneous Binding Assay for Thyroxine, Part II", Bioconj. Chem., in press, 2000. 
14. J. C. Lewis and S. Daunert, "Bioluminescence Immunoassay for Thyroxine Employing Genetically Engineered Mutant Aequorins Containing Unique Cysteine Residues", Anal. Chem., in press 2000

15. J. C. Lewis and S. Daunert, "Photoproteins as Labels in Binding Assays", Fres. J. Anal. Chem., invited contribution, in press, 20000.

16. S. K. Deo, J. C. Lewis, and S. Daunert, "Bioluminescence Detection of Enzymatic Bond Breaking by Using Recombinant Aequorin: Implications in High Throughput Screening", Anal: Biochem., in press, 2000.

17. R. Conover, A. Grosvenor, A. J. Feltus, K. Anderson, and S. Daunert, "Miniaturization of Competitive Binding Assays Using Microfabricated Arrays of pL-Vials", Anal. Chem., in press, 2000.

18. J. C. Lewis, S. Ramanathan, S. Matveev, C. M. Ensor, and S. Daunert, "Extracellular Expression of Recombinant Photoproteins in E. coli", J. Biolum., submitted (1999).

19. A. Grosvenor, K. Anderson, and S. Daunert, "Detection of Biotin in Single Cells Based on the Bioluminescence of the Photoprotein Aequorin", Anal. Chem., to be submitted (2000).

20. U. Desai, J. Wininger-Melton, J. C. Lewis, S. Ramanathan and S. Daunert, "Using EpitopeAequorin Conjugate Recognition in Immunoassays for Complex Proteins", Bioconj. Chem., to be submitted (2000).

21. A. J. Feltus, N. G. Hentz and S. Daunert, "Class-Selective Post-column Reaction Detection Method for Capillary Electrophoresis Based on the Streptavidin-Biotin Interaction", Part I, J. Chromatrogr., to be submitted (2000).

22. A. J. Feltus, N. G. Hentz and S. Daunert, "Single Cell detection of Biotin Coupling Capillary Electrophoresis with a Fluorescence Binding Assay", Part II, J. Chromatrogr., to be submitted (2000).

23. P. M. Douglass, L. L. E. Salins and S. Daunert "A Novel Approach to Drug Detection: Calmodulin as the Detection Element for Phenothiazines and Tri-Cyclic Antidepressants", Anal. Chem., to be submitted (1999).

24. J. Wang and S. Daunert, "Fusions of a Single Chain Antibody and Photoproteins in the Bioluminescence Detection of the Salmonella Antigen", Anal. Biochem., to be submitted (1999). 